\title{
Evolution of interstellar dust with Herschel. First results in the photodissociation regions of NGC 7023 ${ }^{\star}$
}

\author{
A. Abergel ${ }^{1}$, H. Arab ${ }^{1}$, M. Compiègne ${ }^{14}$, J. M. Kirk ${ }^{3}$, P. Ade ${ }^{3}$, L. D. Anderson ${ }^{2}$, P. André ${ }^{4}$, J.-P. Baluteau ${ }^{2}$, \\ J.-P. Bernard ${ }^{7}$, K. Blagrave ${ }^{14}$, S. Bontemps ${ }^{8}$, F. Boulanger ${ }^{1}$, M. Cohen ${ }^{9}$, P. Cox ${ }^{10}$, E. Dartois ${ }^{1}$, G. Davis ${ }^{11}$, R. Emery ${ }^{6}$, \\ T. Fulton ${ }^{12}$, C. Gry ${ }^{2}$, E. Habart ${ }^{1}$, M. Huang ${ }^{11}$, C. Joblin ${ }^{7}$, S. C. Jones ${ }^{16}$, G. Lagache ${ }^{1}$, T. Lim ${ }^{6}$, S. Madden ${ }^{4}$, \\ G. Makiwa ${ }^{16}$, P. Martin ${ }^{14}$, M.-A. Miville-Deschênes ${ }^{1}$, S. Molinari ${ }^{15}$, H. Moseley ${ }^{17}$, F. Motte ${ }^{4}$, D. Naylor ${ }^{16}$, \\ K. Okumura ${ }^{4}$, D. Pinheiro Gonçalves ${ }^{14}$, E. Polehampton ${ }^{16,6}$, J. Rodon ${ }^{2}$, D. Russeil ${ }^{2}$, P. Saraceno ${ }^{15}$, M. Sauvage ${ }^{4}$, \\ S. Sidher ${ }^{6}$, L. Spencer ${ }^{16}$, B. Swinyard ${ }^{6}$, D. Ward-Thompson ${ }^{3}$, G. J. White ${ }^{6,18}$, and A. Zavagno ${ }^{2}$
}

(Affiliations are available in the online edition)

Received 31 March 2010 / Accepted 10 May 2010

\begin{abstract}
Context. In photodissociation regions (PDRs), the physical conditions and the excitation evolve on short spatial scales as a function of depth within the cloud, providing a unique opportunity to study how the dust and gas populations evolve with the excitation and physical conditions. The mapping of the PDRs in NGC 7023 performed during the science demonstration phase of Herschel is part of the "Evolution of interstellar dust" key program. The goal of this project is to build a coherent database on interstellar dust emission from diffuse clouds to the sites of star formation. Aims. We study the far-infrared/submillimeter emission of the PDRs and their fainter surrounding regions. We combine the Herschel and Spitzer maps to derive at each position the full emission spectrum of all dust components, which we compare to dust and radiative transfer models in order to learn about the spatial variations in both the excitation conditions and the dust properties.

Methods. We adjust the emission spectra derived from PACS and SPIRE maps using modified black bodies to derive the temperature and the emissivity index $\beta$ of the dust in thermal equilibrium with the radiation field. We present a first modeling of the NGC 7023-E PDR with standard dust properties and abundances.

Results. At the peak positions, a value of $\beta$ equal to 2 is compatible with the data. The detected spectra and the spatial structures are strongly influenced by radiative transfer effects. We are able to reproduce the spectra at the peak positions deduced from Herschel maps and emitted by dust particles at thermal equilibrium, and also the evolution of the spatial structures observed from the near infrared to the submillimeter. On the other hand, the emission of the stochastically heated smaller particles is overestimated by a factor $\sim 2$.
\end{abstract}

Key words. dust, extinction - photon-dominated region (PDR) - evolution - submillimeter: ISM

\section{Introduction}

The motivation behind the "Evolution of interstellar dust" key program is to explore with Herschel (Pilbrat et al. 2010) the far-infrared (FIR) to submillimeter (submm) emission properties of dust particles in a wide range of regions within our Galaxy, from very diffuse clouds to sites of star formation and protostars. Photometric data taken with SPIRE (Griffin et al. 2010) and PACS (Poglitsch et al. 2010) are complemented with spectroscopy using the FTS of SPIRE and PACS to derive the physical conditions of the gas from the lines of [C I], the high-level rotational lines of $\mathrm{CO}$, and the major cooling lines of [C II] and [O I]. This project is coordinated with the Gould Belt survey (André et al. 2010) and HOBYS (Motte et al. 2010).

Around one third of the observing time of our project is dedicated to photodissociation regions (PDRs) to study how their dust populations and gas content evolve with the excitation and physical conditions. Our sample of PDRs covers a variety of

* Herschel is an ESA space observatory with science instruments provided by European-led Principal Investigator consortia and with important participation from NASA. geometries and spans a wide range of both intensity and hardness of the radiation field.

This paper presents SPIRE and PACS mapping of the reflection nebula NGC 7023, which contains three PDRs illuminated by the Herbig B3 star HD 200775 (Rogers et al. 1995) located at $430 \mathrm{pc}$ (van den Ancker 1997, at this distance $1^{\prime}=0.125 \mathrm{pc}$ ). The three PDRs (NW, E, and S) lies at $\sim 40^{\prime \prime}$ northwest, $\sim 70^{\prime \prime}$ south and $\sim 170^{\prime \prime}$ east of the star, respectively. As discussed by Gérin et al. (1998), NGC 7023 consists of a sheet of dense material in which the star was born, blowing away much of the surrounding gas. The three PDRs at the edges of the remaining material are viewed approximately edge-on. NGC 7023 has been observed extensively in the radio (e.g., Gerin et al. 1998), in $\mathrm{H}_{2}$ lines (Lemaire et al. 1999) and in the visible (e.g., Berné et al. 2008; Witt et al. 2006). Several infrared (IR) features were discovered with ISO and Spitzer (Cesarsky et al. 1996; Werner et al. $2004)$, in addition to strong variations in the 5-35 $\mu \mathrm{m}$ spectra explained by photo-chemical processing of the very small particles (Abergel et al. 2002; Rapacioli et al. 2006; Berné et al. 2007). We can now study with Herschel the big grain component, which contains most of the dust mass. 


\section{Interstellar dust}

Interstellar dust comprises several components. The smallest grains are carbonaceous particles (polycyclic aromatic hydrocarbons PAHs, and "very small grains" VSGs) containing $\sim 10$ 1000 carbon atoms. They are stochastically heated, and emit most of their thermal energy below $\sim 60 \mu \mathrm{m}$. The big grains (BGs) have sizes of $\sim 100 \mathrm{~nm}$ and consist of amorphous silicates and carbon. They are in thermal equilibrium with the interstellar radiation field (ISRF). Their emission spectrum peaks in the sub-mm, and can be modeled as a modified black body $I_{\lambda}=\epsilon_{\lambda_{0}}\left(\frac{\lambda}{\lambda_{0}}\right)^{-\beta} B_{\lambda}(T)$, where $\epsilon_{\lambda_{0}}$ is the emissivity at $\lambda_{0}, \beta$ the spectral index, $B_{\lambda}$ the Planck function, and $T$ the temperature.

Interstellar dust can be described by various models. In the post-Spitzer/pre-Herschel era, the silicate-graphite-PAH model of Draine \& Li (2007) and the silicate-amorphous carbon-PAH model of Compiègne et al. (in prep.) account consistently for the observations (extinction, scattering, emission, depletions). Both models consider separate silicate and carbonaceous particles, which inferred to be present because the $9.7 \mu \mathrm{m}$ band is polarized but the $3.4 \mu \mathrm{m} \mathrm{C-H} \mathrm{bond} \mathrm{stretch} \mathrm{is} \mathrm{not} \mathrm{(e.g.,} \mathrm{Chiar} \mathrm{et} \mathrm{al.}$ 2006). Silicate-core/carbonaceous-mantle models (e.g., Désert et al. 1990) and composite models with aggregates (e.g., Zubko et al. 2004) have also been proposed.

Up to now, the sub-mm emission has been measured by very few experiments. DIRBE and FIRAS on board COBE produced all-sky maps of resolutions $40^{\prime}$ and $7^{\circ}$, respectively. The dust temperature is found to be on average $\sim 17.5 \mathrm{~K}$ (with $\beta=2$ ) in the diffuse atomic medium (Boulanger et al. 1996) and to be lower in molecular clouds with no embedded bright stars (Lagache et al. 1998). Small patches of bright molecular clouds have been observed in more detail from the ground by the JCMT (Johnstone et al. 2006), and from the balloon borne experiments PRONAOS (Ristorcelli et al. 2006) and Archeops (Désert et al. 2008). At low temperatures $(T<30 \mathrm{~K})$, the dust optical properties appear to change significantly in terms of absolute value of the emissivity $\epsilon$ and the spectral index $\beta$. As also seen in laboratory measurements (e.g., Agladze et al. 1996; Mennella et al. 1998; Boudet et al. 2005), the physical processes responsible for these effects probably involve ice mantle formation, grain coagulation, and low-energy structural transformations (e.g., Meny et al. 2007).

\section{Observations}

NGC 7023 was mapped during the science demonstration phase on September 9 and November 112009 by SPIRE and PACS, respectively. For SPIRE, two perpendicular $8^{\prime} \times 8^{\prime}$ large maps were performed with the nominal scan speed $\left(30^{\prime \prime} / \mathrm{s}\right)$, and a repetition of 4 (for a total observing time of $1675 \mathrm{~s}$ ). We use the Level-2 naive maps delivered by the the HSC, with standard corrections for instrumental effects and glitches. The overall absolute flux accuracy is $15 \%$ (Griffin et al. 2010; Swinyard et al. 2010).

For PACS, two perpendicular $10^{\prime} \times 10^{\prime}$ scan maps were performed with the medium scan speed $\left(20^{\prime \prime} / \mathrm{s}\right)$, a scan length of $10^{\prime}$, a cross-scan step of $15^{\prime \prime}$, and a number of scan legs of 41 (total observing time $5166 \mathrm{~s}$ ). For the blue channel, the $70 \mu \mathrm{m}$ filter was selected. The data were processed with HIPE (version 2.3.1). We performed simple projection with second level deglitching. The $1 / \mathrm{f}$ noise components were removed using high pass filtering, with a window size equal to the scan length. Data taken in the two scanning directions were processed independently before averaging. For the bright parts of detected structures, the differences between the two computed maps are below the absolute flux uncertainties, within $10 \%$ and $20 \%$ in the blue and red bands, respectively (Poglitsch et al. 2010; Swinyard et al. 2010). On the other hand, the faint regions around bright structures appear to exhibit some artifacts.

The processed maps are shown with Spitzer maps from Kirk et al. (2009) in Fig. 1. For the quantitative analysis, we subtracted a constant background taken around the position $\alpha_{2000}=$ $21^{\mathrm{h}} 0^{\mathrm{m}} 49^{\mathrm{s}}, \delta_{2000}=68^{\circ} 07^{\prime} 14^{\prime \prime}$. We also degraded all maps to match the SPIRE resolution at $500 \mu \mathrm{m}$ (with the preliminary assumption of Gaussian beams).

The PDRs correspond to bright filaments at long wavelengths because of a combination of a steep increase in the column density and the extinction of incident radiation. The distance from the illuminating star to the peak of the brightness profiles across the PDRs increases with increasing wavelength, as illustrated in Fig. 2 for NGC 7023-E. This is also observed in Fig. 1: the size of the detected images increases with increasing wavelengths (this is not due to an increase in the beam size).

\section{Spectrum of the BG component}

The PACS and SPIRE data allows for the first time the measurement of the spectrum of the BG component on both sides of the spectral peak. The spectra obtained at different positions can be adjusted with a modified black body to derive $T$ and $\beta$. In this first result paper, we focus on the brightest positions (at $250 \mu \mathrm{m}$ ) of the three PDRs (Fig. 3). Within the error bars, the three spectra are reasonably adjusted with a fixed value of $\beta=2$, as for the average spectrum of the diffuse ISM. A free value of $\beta$ provides slightly superior fits to the data in NGC $7023 \mathrm{NW}$ and -S, with $\beta=2.3$ and 2.6 , respectively.

\section{Interpretation of the emission spectrum}

At each position in the maps, the combination of Spitzer and Herschel data provides an emission spectrum for all dust components, as illustrated in Fig. 5. We used the "DUSTEM" model of Compiègne et al. (in prep.) to compute the emission spectrum at the $250 \mu \mathrm{m}$ peak position of NGC 7023-E using the reference dust population, which allowed the reproduction of the observed extinction and emission for the diffuse high Galactic latitude ISM. We followed Gerin et al. (1998) in estimating the UV radiation field to be $\sim 250$ times the strength of the radiation field in the solar neighborhood of Mathis et al. (1983). In Fig. 5, we see that the computed spectrum for the BG component (normalized to the data at $70 \mu \mathrm{m}$ ) fails to reproduce the observed spectrum, peaking at shorter wavelengths (black line in Fig. 5).

To quantify the radiative transfer effects, we used the model described in Compiègne et al. (2008) coupled with DUSTEM. The PDR was represented by a plane-parallel slab that is assumed to have an arbitrary density profile $n_{H}(z)$ illuminated by the IRSF (Mathis et al. 1983) added to the stellar radiation field. This transfer model accounts for the effect of scattering by separating forward (i.e., transmitted) from backward scattering. The dust heating by IR photons emitted by dust is also considered.

The illuminated side and the rear side of the density profile were constrained by the observations. We used the symmetric density profile shown in Fig. 4. The three parameters were adjusted $\left(z_{0}=0.18 \mathrm{pc}, n_{0}=3 \times 10^{4} \mathrm{~cm}^{-3}\right.$, and $\left.\alpha=2.8\right)$ to reproduce the brightness profiles at all wavelengths (Fig. 2). We also adjusted the length of the PDR along the line of sight $\left(L_{\mathrm{PDR}}=0.65 \mathrm{pc}\right)$ to match the observed brightness at $70 \mu \mathrm{m}$.

The computed spectrum (Fig. 5) reproduces the PACS and SPIRE data for the BGs, but overestimates by a factor $\sim 2$ the emission at 3.6 to $24 \mu \mathrm{m}$ which is produced by PAHs and VSGs. This result may indicate that the relative abundance of the small 


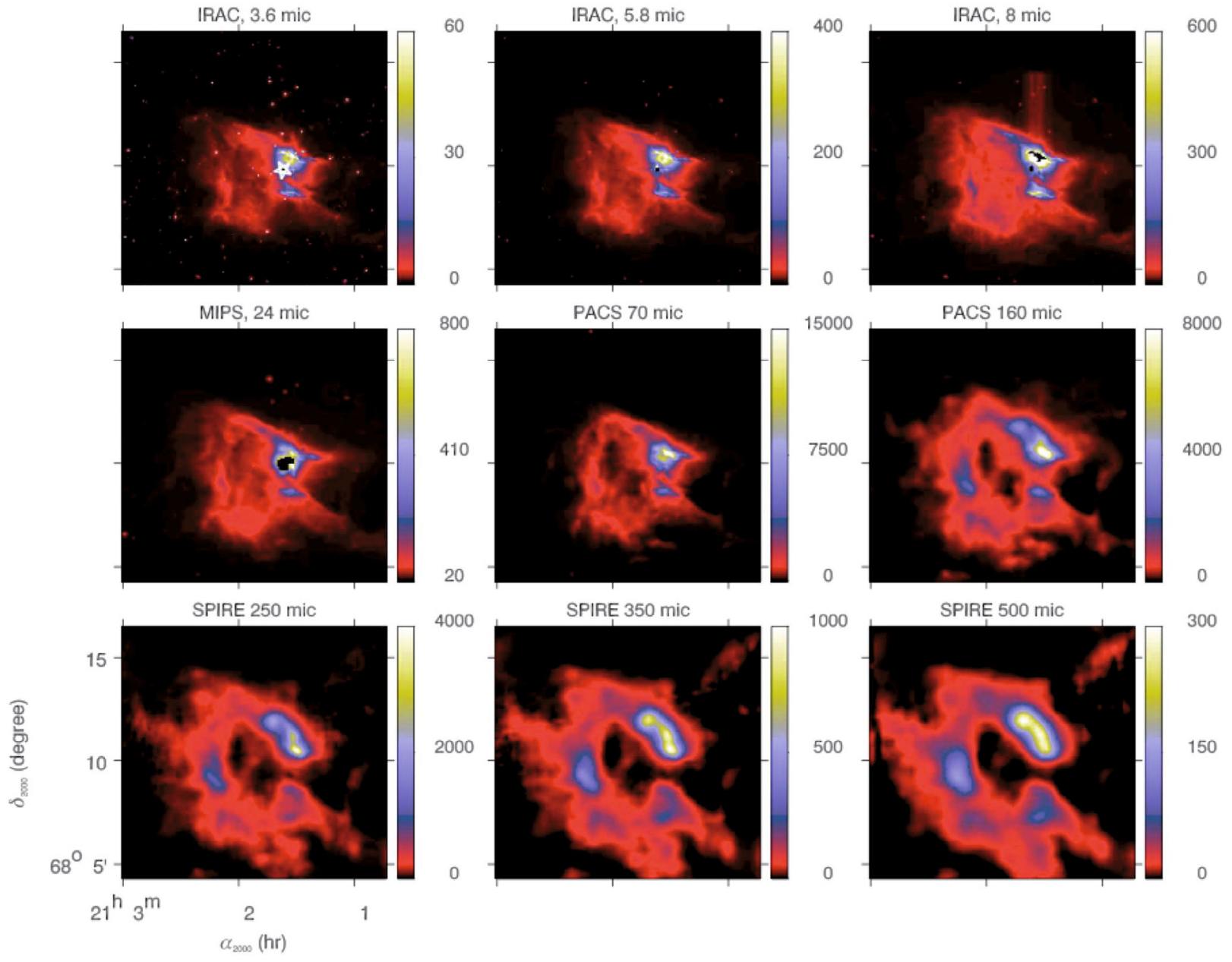

Fig. 1. NGC 7023 maps obtained with Spitzer (from Kirk et al. 2009) and Herschel. Units of the color bars are MJy/sr. The position of the illuminating star is seen on the $3.6 \mu \mathrm{m}$ map, in addition to the position of the brightness profile of Fig. 2.

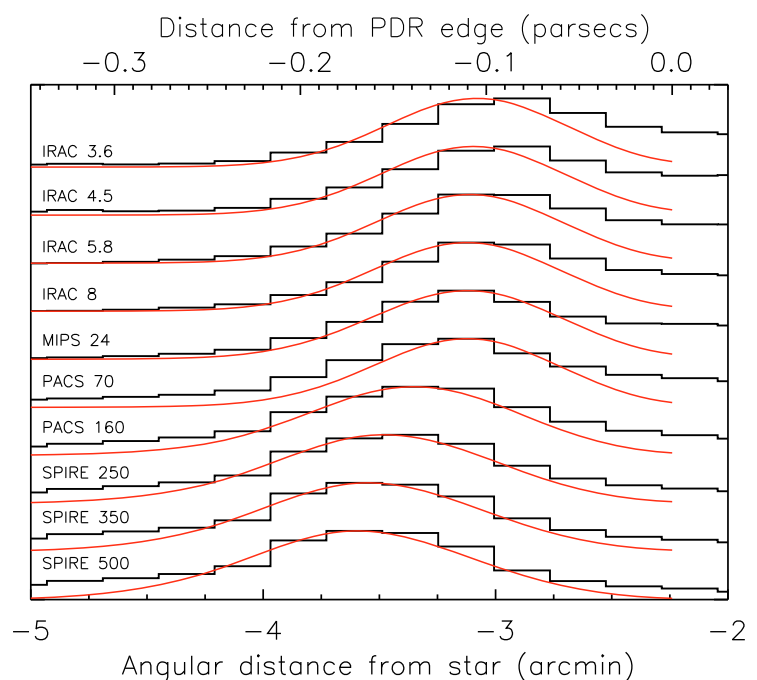

Fig. 2. Normalized brightness profiles across NGC 7023-E along the cut shown in Fig. 1. Black: data at the SPIRE resolution at $500 \mu \mathrm{m}$. Red: outputs of our radiative transfer model (see Sect. 5).

dust particles is lower, or the absolute emissivity of the BGs is higher, than in the diffuse high Galactic latitude ISM, as already observed in dense molecular clouds (e.g., Stepnik et al. 2003). We also derived the length of the PDR along the line of sight to be $0.65 \mathrm{pc}$, which is relatively large compared to the width of the

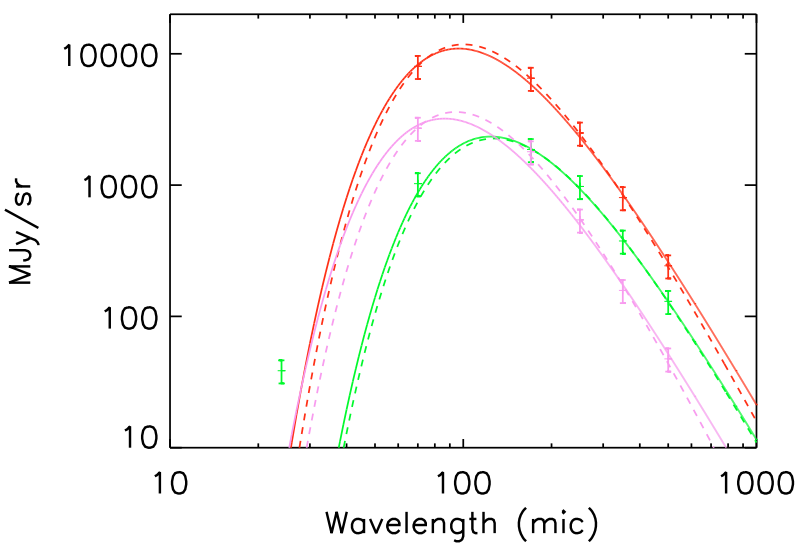

Fig. 3. Spectra at the $250 \mu \mathrm{m}$ peak positions of the three PDRs NGC 7023-NW (red), -S (purple) and -E (green). Fits with $\beta=2$ (solid lines): $T=30.0 \mathrm{~K}, 33.5 \mathrm{~K}$ and $23.3 \mathrm{~K}$. Fits with free values of $T$ and $\beta$ (dashed lines): $T=27.1 \mathrm{~K}, 27.2 \mathrm{~K}$, and $22.1 \mathrm{~K}$ with $\beta=2.3,2.6$, and 2.1. For NGC 7023-E, data points at $70 \mu \mathrm{m}$ are excluded from the fit, since the emission from stochastically heated small particles can contribute, as shown in Sect. 5 (in the two other PDRs, the excitation is higher, so the emission of dust at thermal equilibrium is dominant at $70 \mu \mathrm{m}$ ).

filament $(\sim 0.15 \mathrm{pc})$. A model with a higher BG emissivity infers a length that is comparable to the width.

We conclude that radiative transfer effects can explain the spatial shifts between the brightness profiles (Fig. 2), and the 


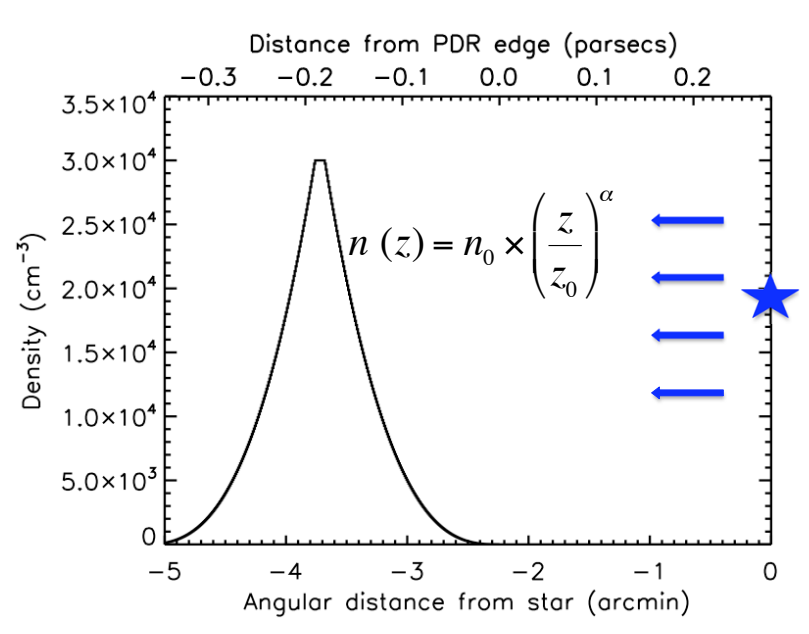

Fig. 4. Density profile taken to model the NGC 7023-E PDR.

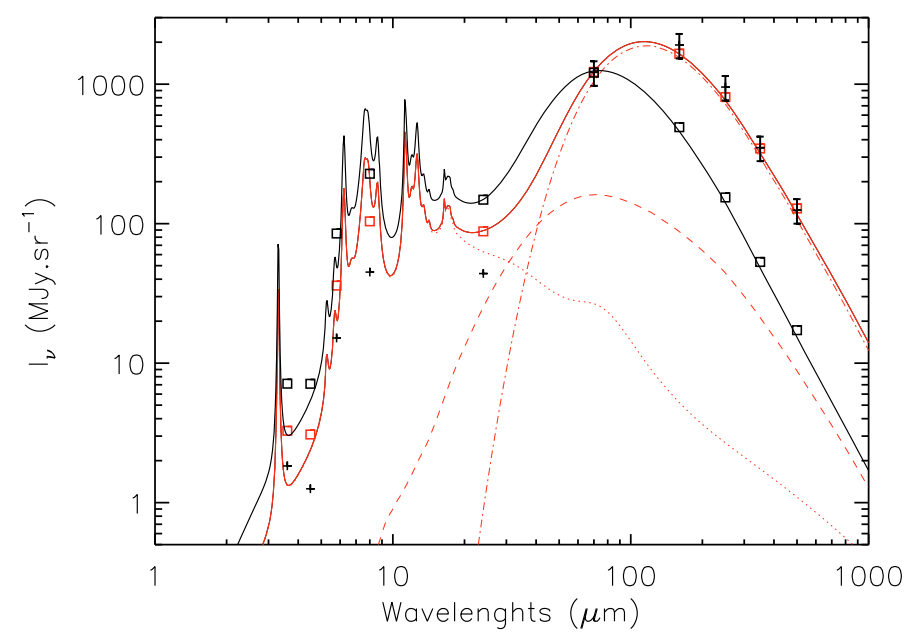

Fig. 5. Crosses: Spitzer and Herschel data points at the $250 \mu \mathrm{m}$ peak position of NGC 7023-E. The absolute uncertainties for IRAC and MIPS data are $1.5 \%$ and $4 \%$, respectively (Kirk et al. 2009). Solid black line: emission spectrum computed at the same position with the DUSTEM model of Compiègne et al. (in prep.) (squares: color-corrected brightnesses in the different filters). Solid red line: the same with our radiative transfer model. The total spectrum is the sum of the spectra computed for the PAH, VSG and BG components (dotted, dashed, and dasheddotted lines, respectively).

increase in the size of the detected images with increasing wavelength (Fig. 1). We can also explain why the $70 \mu \mathrm{m}$ map is more comparable to the Spitzer than the Herschel maps. The $70 \mu \mathrm{m}$ PACS bandpass is on the left-hand side of the spectral peak of the BGs (Fig. 5), with a minor contribution from the VSGs, so the measured emission strongly depends on the incident radiation field, as for the emission at shorter wavelength produced by stochastically heated dust particles. The $70 \mu \mathrm{m}$ map therefore resembles the Spitzer maps detecting the PAH and VSG emission, while the $160 \mu \mathrm{m}$ map is more comparable to the SPIRE maps studying the Rayleigh-Jean part of the spectrum.

\section{Conclusions}

For the first time, we have detected the FIR/submm filaments at the PDRs of NGC 7023. We have demonstrated that the emission spectra derived from SPIRE and PACS maps can be adjusted with modified black bodies. Our first results at the peak positions have indicated that the value of $\beta$ equal to 2 found in the diffuse atomic medium is compatible with the data. Spatial variations in $\beta$ have also been found, but the data processing must be stabilized before any quantitative analysis is possible. The combination of SPIRE, PACS, and Spitzer maps has provided full emission spectra of all dust particles that have been compared to dust and radiative transfer models. Our first results for NGC 7023-E illustrate the dramatic influence of radiative transfer on the spatial structures observed at long wavelengths. The next step is to combine imaging and spectroscopic Herschel data to achieve deeper insight into both the dust and gas components.

Acknowledgements. SPIRE has been developed by a consortium of institutes led by Cardiff Univ. (UK) and including Univ. Lethbridge (Canada); NAOC (China); CEA, LAM (France); IFSI, Univ. Padua (Italy); IAC (Spain); Stockholm Observatory (Sweden); Imperial College London, RAL, UCL-MSSL, UKATC, Univ. Sussex (UK); Caltech, JPL, NHSC, Univ. Colorado (USA). This development has been supported by national funding agencies: CSA (Canada); NAOC (China); CEA, CNES, CNRS (France); ASI (Italy); MCINN (Spain); SNSB (Sweden); STFC (UK); and NASA (USA). PACS has been developed by a consortium of institutes led by MPE (Germany) and including UVIE (Austria); KUL, CSL, IMEC (Belgium); CEA, OAMP (France); MPIA (Germany); IFSI, OAP/AOT, OAA/CAISMI, LENS, SISSA (Italy); IAC (Spain). This development has been supported by the funding agencies BMVIT (Austria), ESAPRODEX (Belgium), CEA/CNES (France), DLR (Germany), ASI (Italy), and CICT/MCT (Spain). We thank Lori Allen for providing improved IRAC and MIPS maps of NGC 7023 taken from the "Spitzer Gould Belt legacy program", and Karin Dassas for her help in the PACS data processing.

\section{References}

Abergel, A., Bernard, J. P., Boulanger, F., et al. 2002, A\&A, 389, 239 Agladze, N. I., Sievers, A. J., Jones, S. A., et al. 1996, ApJ, 462, 1026 André, Ph., et al. 2010, A\&A, 518, L102

Berné, O., Joblin, C., Deville, Y., et al. 2007, A\&A, 469, 575

Berné, O., Joblin, C., Rapacioli, M., et al. 2008, A\&A, 479, L41

Boulanger, F., Abergel, A., Bernard, J.-Ph., et al. 1996, A\&A, 312, 256

Boudet, N., Mutschke, H., Nayral, C., et al. 2005, ApJ, 633, 272

Cesarsky, D., Lequeux, J., Abergel, A., et al. 1996, A\&A, 315, 305

Chiar, J. E., Adamson, A. J., Whittet, D. C. B., et al. 2006, ApJ, 651, 268

Compiègne, M., Abergel, A., Verstraete, L., et al. 2008, A\&A, 491, 797

Désert, F.-X., Boulanger, F., \& Puget, J. L. 1990, A\&A, 237, 215

Désert, F.-X., Macias-Perez, J. F., Mayet, F., et al. 2008, A\&A, 481, 411

Draine, B. T., \& Li, A. 2007, ApJ, 657, 810

Dupac, X., Bernard, J.-P., Boudet, N., et al. 2003, A\&A, 404, L11

Gerin, M., Phillips, T. G., \& Keene, J., et al. 1998, ApJ, 500, 329

Griffin, M. J., et al. 2010, A\&A, 518, L3

Johnstone, D., Matthews, H., \& Mitchell, G. F. 2006, ApJ, 639, 259

Kirk, J. M., Ward-Thompson, D., Di Francesco, J., et al. 2009, ApJS, 185, 198 Lagache, G., Abergel, A., Boulanger, F., \& Puget, J. L. 1998, A\&A, 333, 709

Lemaire, J. L., Field, D., \& Maillard, J. P. 1999, A\&A, 349, 253

Mathis, J. S., Mezger, P. G., \& Panagia, N. 1983, A\&A, 128, 212

Mennella, V., Brucato, J. R., Colangeli, L., et al. 1998, ApJ, 496, 1058

Meny, C., Gromov, V., Boudet, N., et al. 2007, A\&A, 468, 171

Motte, F., et al. 2010, A\&A, 518, L77

Pilbratt, G. L., et al. 2010, A\&A, 518, L1

Poglitsch, A., et al. 2010, A\&A, 518, L2

Rapacioli, M., Joblin, C., \& Boissel, P. 2005, A\&A, 429, 193

Ristorcelli, I., Serra, G., Lamarre, J.-M., et al. 1998, ApJ, 496, 267

Rogers, C., Heyer, M. H., \& Dewdney, P. E. 1995, ApJ, 442, 694

Stepnik, B., Abergel, A., Bernard, J.-P., et al. 2003, A\&A, 398, 551

Swinyard, B. M. et al. 2010, A\&A, 518, L4

van den Ancker, M. E., Thé, P. S., Tjin A Djie, H. R. E., et al. 1997, A\&A, 324, L33

Werner, M. W., Uchida, K. I., Sellgren, K., et al. 2004, ApJS, 154, 309

Witt, A. N., Gordon, K. D., Vijh, U. P., et al. 2006, ApJ, 636, 303

Zubko, V., Dwek, E., \& Arendt, R. G. 2004, ApJS, 152, 211

Page 5 is available in the electronic edition of the journal at http://www . aanda.org 
1 Institut d'Astrophysique Spatiale, UMR 8617, CNRS/Université Paris-Sud 11, 91405 Orsay, France

e-mail: alain.abergel@ias.u-psud.fr

2 Laboratoire d'Astrophysique de Marseille (UMR 6110 CNRS \& Université de Provence), 38 rue F. Joliot-Curie, 13388 Marseille Cedex 13, France

3 Department of Physics and Astronomy, Cardiff University, Cardiff, UK

4 CEA, Laboratoire AIM, Irfu/SAp, Orme des Merisiers, 91191 Gif-sur-Yvette, France

5 Dept. of Physics \& Astronomy, University College London, Gower Street, London WC1E 6BT, UK

6 The Rutherford Appleton Laboratory, Chilton, Didcot OX11 0QX, UK

7 CESR, Université de Toulouse (UPS), CNRS, UMR 5187, 9 avenue du colonel Roche, 31028 Toulouse Cedex 4, France

8 CNRS/INSU, Laboratoire d'Astrophysique de Bordeaux, UMR 5804, BP 89, 33271 Floirac Cedex, France
9 University of California, Radio Astronomy Laboratory, Berkeley, 601 Campbell Hall, US Berkeley CA 94720-3411, USA

${ }^{10}$ Institut de Radioastronomie Millimétrique (IRAM), 300 rue de la Piscine, 38406 Saint-Martin-d'Hères, France

11 National Astronomical Observatories (China)

12 Blue Sky Spectrosocpy Inc, Lethbridge, Canada

13 Department of Physics and Astronomy, University of British Columbia, Vancouver, Canada

14 Canadian Institute for Theoretical Astrophysics, Toronto, Ontario, M5S 3H8, Canada

15 Istituto di Fisica dello Spazio Interplanetario, INAF, Via del Fosso del Cavaliere 100, 00133 Roma, Italy

16 Institute for Space Imaging Science, University of Lethbridge, Lethbridge, Canada

17 NASA - Goddard SFC, USA

18 Department of Physics \& Astronomy, The Open University, Milton Keynes MK7 6AA, UK 